\title{
PHASE AND IMAGE RECONSTRUCTION FROM PUPIL-PLANE OBSERVATIONS IN THE NEAR INFRARED:
}

\author{
INTEGRATION OF THE PHASORS
}

\author{
J.-L. MONIN ${ }^{1}$, N. AGEORGES ${ }^{1}$, L. DESBAT ${ }^{2}$ \\ and \\ C. PERRIER ${ }^{1}$ \\ 1. Observatoire de Grenoble, BP 53X, 38041 Grenoble Cedex, France \\ 2. TIMB-TIM3, UJF-CNRS-CHUG, Faculté de Médecine, 38706 Grenoble Cedex, France \\ january 1993
}

\begin{abstract}
A new method to reconstruct the phase of bidimensional interferograms, obtained through pupil-plane interferometry is presented. We compute the average complex phasor components of the cross-spectrum on a data set to reconstruct the original unperturbed phase. We present preliminary results on simulated images which visibility phases are distorted using a model of atmospheric perturbed wavefronts.
\end{abstract}

Key words: Interferometry - Infrared - IR Arrays - Image reconstruction

\section{Introduction}

The 1 to $5 \mu \mathrm{m}$ range is well suited to study the properties of stars and their immediate surroundings, eg. multiple systems, protostellar disks, envelopes, etc. These phenomena show up at linear scales of the order of a few stellar radii, corresponding to angular size ranging from 0.01 to 1 arcsec at the nearest molecular clouds distances. This resolution is within the range of a $4-10$ meter telescope at $\lambda \approx 1 \mu \mathrm{m}$, but atmospheric turbulence drastically limits the resolution of ground-based infrared observations to 1 arcsec or more because of the phase shifts induced to the incident wavefront during its propagation through the atmospheric boiling.

Several interferometric techniques have been developped to recover the initial diffraction-limited angular resolution from perturbed images / wavefronts. Except for Adaptative Optics, all interferometric techniques are a posteriori techniques and rely on computations of the modulus and phase of the observed visibility. In the image plane, the Speckle interferometry has been shown to allow full image reconstruction at the diffraction-limited resolution of the telescope (Weigelt, 1989). Mariotti et al. (1992) have described a method based on pupil plane interferometry in the near infrared. This method was previously successfully tested in the visible by Roddier \& Roddier (1979, 1989 and reference therein). The advantage of this method is that its associated Modulation Transfer Function is not affected by wavefront phase errors and remains at a high value $(\approx 100 \%)$ up to the cutoff frequency of the telescope. In pupil plane interferometry, one directly measures the components of the complex visibility function. Unlike the modulus, the phase can not be averaged over the successive interferograms because shifts exceeding $2 \pi$ can be induced on the wavefront during its propagation through the atmosphere. This problem has received considerable attention in the last decade (for a review, see e.g. Chelli, 1989). Monin et al. (1992) presented a method to recover the phase of the complex visibility, using spatial gradients of the instantaneous measured phase. Their method was derived from the KnoxThompson algorithm, using the correlation of the incoming wavefront between adjacent spatial frequencies. They computed the solution of the equation : $\nabla \phi=d$. 
In this paper, we report on preliminary results on an improvement of this method, working on the complex phasors instead of the phase. This method has been tested on simulated data. As the main perturbation during the propagation occurs on the phase and not on the modulus of the visibility, we suppose the latter to be known independently.

\section{Theory}

\subsection{INITIAL DATA}

We suppose that the complex components of the visibility of the image are known as $X$ and $Y$ :

$$
X=|\gamma| \cos (\Phi)=|\gamma| \cos \left(\phi+\phi_{a}+\phi_{o}\right) ; Y=|\gamma| \sin (\Phi)=|\gamma| \sin \left(\phi+\phi_{a}+\phi_{o}\right)
$$

where $\phi$ represents the object's phase (to be determined), $\phi_{a}$ the random phase introduced by the atmosphere and $\phi_{o}$ the phase related to the aberrations (assumed constant) of the optical set-up. Even if the initial phase of the object is perturbed by the atmospheric boiling, these perturbations are correlated from one point to another, provided the distance between the two points of the wavefront is sufficiently small. Following this idea, the average complex cross spectrum $C=|C| e^{i \phi_{C}}$ can be computed :

$$
C(\mathbf{f}, \Delta \mathbf{f})=\left\langle\gamma^{\prime}(\mathbf{f}) \gamma^{\prime *}(\mathbf{f}+\Delta \mathbf{f})\right\rangle=\left\langle|\gamma(\mathbf{f}) \| \gamma(\mathbf{f}+\Delta \mathbf{f})| e^{i \nabla \Phi}\right\rangle
$$

Where \langle\rangle represents an average over numerous successive interferograms. Assuming that the term $|\gamma(f)||\gamma(f+\Delta f)|$ does not vary over the pupil, or that the modulus $|\gamma|$ is independently known (see Mariotti et al., 1992), equation 2 reduces to a phasor equation : $e^{i \nabla \Phi}=d$, where $d$ represents our data and $\Phi$ the unknown phase. During the averaging process, the random atmospheric term $\phi_{a}$ vanishes and one is left with $\phi+\phi_{o}$. The aberrations can be calibrated out against a reference star, so that we will hereafter only consider $\phi$ in our equations.

\subsection{Phase integration}

Just as in Monin et al. (1992), the operator $\nabla$ associates to $n \times n$ phase "images" their discrete gradient in both directions in the Fourier plane. The operator involved in equation 2 associates the vector $e^{i \nabla \phi} \in \mathbb{Z}^{2 * n *(n-1)}$ to the vector $\phi \in \mathbb{R}^{n^{2}}$. Thus we want to compute the best estimation of the phase $\phi$ of the object from the data $d$ i.e. we want to solve the following problem :

$$
\min _{\phi \in \mathbb{R}^{n^{2}}}\left\|e^{i \nabla \phi}-d\right\|^{2}
$$

For that purpose, we used a Gauss-Newton method (Lannes, 1990 and reference therein) based on the local linearisation of the functional :

$$
e^{i \nabla(\phi+h)}=e^{i \nabla \phi}+i e^{i \nabla \phi} \nabla h+o(h)
$$

The Gauss-Newton method is iterative. At each iteration $\phi^{k+1}:=\phi^{k}+h^{k}$ with $h^{k}$ minimising the following quadratic form :

$$
\min _{h \in \mathbb{R}^{n^{2}}}\left\|i e^{i \nabla \phi} \nabla h-r^{k}\right\|^{2}
$$


with

$$
r^{k}=d-e^{i \nabla \phi^{k}}
$$

The vector $h$ can be computed with the (same) Conjugate Gradient method used in Monin et al. (1992).

\section{Numerical simulations}

We have tested the method on two dimensional simulated data. Starting from an image, we compute its visibility and save its modulus for later processing. We use a wavefront simulation program written by $\mathrm{N}$. Roddier (1990) to perturbe the phase. The visibility phasor is computed as $x_{o}+i y_{o}$, with $\sqrt{x_{o}^{2}+y_{o}^{2}}=1$. The simulated wavefronts are computed as a phase $\varphi_{a}$, and the corresponding perturbating phasor as $x_{a}+i y_{a}=\cos \left(\varphi_{a}\right)+i \sin \left(\varphi_{a}\right)$. Then we get the perturbed data : $X=x_{o} x_{a}-y_{o} y_{a} ; Y=x_{o} y_{a}+y_{o} x_{a}$, and $X$ and $Y$ are used to compute the cross spectrum $d(f, \Delta f)$, with modulus equal 1 and we solve (3). All our simulation computations have been performed using the sofware package IDL (trademark of Research systems Inc.) and the GRAPHIC software package developped at the Groupe d'Astrophysique de Grenoble.

\section{Results}

The method has been tested on various kinds of objects. We show here the results obtained on a triple star with identical unresolved components separated by 5 elements of resolution (see figure 1 and its caption for details). We used a pupil size of radius 18 pixels. This allows simulation with $\mathrm{D} / r_{o}$ up to 18 . All our computations were made using $\mathrm{D} / r_{0}=$ 5 corresponding to realistic turbulence conditions on a $4 \mathrm{~m}$ telescope at $2.2 \mu \mathrm{m}$. All our simulations were reconstructed by averaging the cross spectrum over 128 perturbed wavefronts. Indeed, this is a very small number of instantaneous interferograms and we can expect the results to be far better by using ten times this number of frames. As we expect that in the near future every telescope will be equiped with at least a partial adaptative optics correction system, we have removed the tilt on the simulated incoming wavefront.

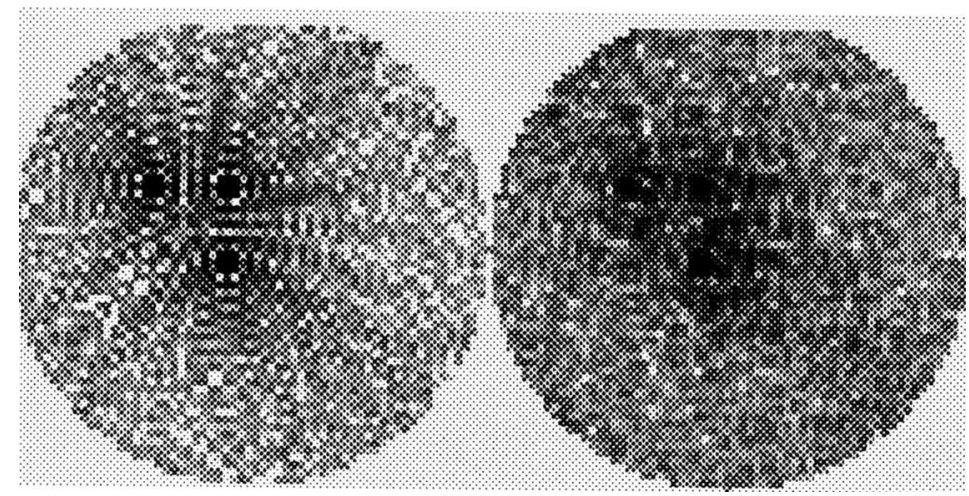

Fig. 1. Simulation and reconstruction of a triple star system. Left : the initial image. Right : reconstructed image obtained after convergence in about 100 iterations. 


\section{Discussion}

One of the main problems in phase reconstruction is the phase measure itself. The wrapping operation forbids direct averaging and the computation of the phase from $\arctan (Y / X)$ like functions introduce spurious values when the $X$ component is at the noise level. It appears that any phase reconstruction procedure has to work on the phasor components (however, see Haniff, 1991, for an alternative nonlinear least squares method that explicitely takes into account the modulo $2 \pi$ nature of the phases). In the simulations presented in this paper, we have avoided any actual computation of the phase. The perturbed wavefront phase information is known with a high signal to noise ratio and immediatly converted into phasor components. All the subsequent operations performed on the 'phase' data are phasor computations. The only questionnable operation is the normalisation of the visibility when its modulus is at the noise level. This is less critical than the $\arctan (Y / X)$ operation because it requires both $X$ and $Y$ to be at the noise level. For marginally resolved objects, this problem is expected to happen on the edges of the pupil (the pupil-plane MTF is almost constant up to the cutoff frequency) and / or at a limited number of points within the pupil, where the phasor can be interpolated. The detailed testing of this method and comparison with the scalar phase reconstruction proposed in Monin et al., (1992) will be presented in a forthcoming paper.

The next step in our phase reconstruction approach will be to work on the complete cross-spectrum equation : $\gamma^{2} e^{i \nabla \phi}=C$, solving it for both the phase and the modulus of the object visibility from its cross-spectrum.

\section{References}

Chelli, A.: 1989, in Alloin D., Mariotti J.-M., ed(s)., Diffraction limited imaging with very large telescopes, NATO ASI Vol. C 274, Cargèse, September 1988 Kluwer, Dordrecht (1989)

Haniff, C.A.: 1991, J.O.S.A. Vol. 8 no 1,134

Lannes, A.: 1990, J.O.S.A. Vol.7 no 3, 500

Mariotti, J. M., Monin, J. L., Ghez, P., Perrier, C. and Zadrozny,A.: 1992, A\&A 255, 462

Monin, J. L., Mariotti, J. M., Ghez, P., Perrier C. and Desbat, L.: 1992, $A \& A$ 260, 510

Roddier, C., Roddier, F.: 1979, Proc. IAU Coll. No49, 175

Roddier, C., Roddier, F.: 1990, in Alloin D., Mariotti J.-M., ed(s)., Diffraction limited imaging with very large telescopes, NATO ASI Vol. C 274, Cargèse, September 1988 Kluwer, Dordrecht (1989)

Roddier, N.: 1990, in Breckinridge J.B., ed(s)., Amplitude and Intensity Spatial Interferometry, Proc. S.P.I.E. Conf. No 1237, Tucson (1990)

Weigelt, G.: 1989, in Alloin D., Mariotti, J.M., ed(s)., Diffraction limited imaging with very large telescopes, NATO ASI Vol. C 274, Cargèse, September 1988

Kluwer, Dordrecht (1989) 